\title{
PARACOMPACTNESS IN PERFECTLY NORMAL, LOCALLY CONNECTED, LOCALLY COMPACT SPACES
}

\author{
DIANE J. LANE
}

\begin{abstract}
It is shown that, under (MA $+\neg \mathrm{CH}$ ), every perfectly normal, locally compact and locally connected space is paracompact.
\end{abstract}

In $[\mathbf{R u}, \mathbf{Z}]$ Rudin and Zenor use the continuum hypothesis $(\mathrm{CH})$ to construct a perfectly normal, separable manifold that is not Lindelöf and is therefore not paracompact. Manifold here means a locally Euclidean Hausdorff space. Rudin has shown recently [Ru] that if Martin's Axiom and the negation of the continuum hypothesis (MA $+\neg \mathrm{CH}$ ) hold, then every perfectly normal manifold is metrizable. In this paper we show that Rudin's technique can be used to obtain a more general result: If (MA $+\neg \mathbf{C H}$ ), then every perfectly normal, locally compact and locally connected space is paracompact. Since locally metrizable paracompact spaces are metrizable, Rudin's theorem follows.

The following theorems will be used.

TheOREM 1 (Z. Szentmiklossy [S]). If $(M A+\neg C H)$, then there is no hereditarily separable, nonhereditarily Lindelöf, compact (locally compact) Hausdorff space.

THEOREM 2 (JuHASZ [J]). If $(M A+\neg C H)$, then there is no hereditarily Lindelöf, nonhereditarily separable compact (locally compact) Hausdorff space.

THEOREM 3 (REED AND ZENOR [R, Z]). Every perfectly normal, locally compact and locally connected subparacompact space is paracompact.

THeOREM 4 (ALSTER AND ZENOR [A, Z]). Every perfectly normal, locally compact and locally connected space is collectionwise normal with respect to discrete collections of compact sets.

The following result was obtained independently by $\mathbf{H}$. Junilla and J. Chaber. A proof can be found in $[\mathbf{C}, \mathbf{Z}]$.

THEOREM 5. A space $X$ is perfect and subparacompact if and only if whenever $\left\{W_{\beta}\right\}_{\beta<\gamma}$ is a well-ordered open cover of $X$, there exists a sequence $\left\{\mathcal{U}_{n}\right\}_{n \in \omega}$ of open covers of $X$ with the property that if $x \in X$, there exists $n \in \omega$ such that $\operatorname{st}\left(x, \mathcal{U}_{n}\right)=$ $\left\{U \in \mathcal{Q}_{n} \mid x \in U\right\}$ is contained in the first member of $\left\{W_{\beta}\right\}_{\beta<\gamma}$ that contains $x$.

Received by the editors May 21, 1979 and, in revised form, January 2, 1980.

1980 Mathematics Subject Classification. Primary 54D18, 54D05; Secondary 03E50, $03 E 35$.

Key words and phrases. Martin's Axiom, subparacompact, paracompact, perfectly normal, locally compact, locally connected, hereditarily separable, hereditarily Lindelö, continuum hypothesis, manifold. 
We prove

THEOREM 6. If $(M A+\neg C H)$, then every perfectly normal, locally compact and locally connected space is paracompact.

Proof. Let $X$ be a perfectly normal, locally compact and locally connected space. Since components of a locally connected space are open, we may assume that $X$ is connected. We prove that $X$ is subparacompact and thus, by Theorem 3, is paracompact.

Since compact subsets of a perfect space are hereditarily Lindelöf, Theorem 2 implies that compact subsets of $X$ are hereditarily separable.

By transfinite induction we choose for each $\alpha<\omega_{1}$ a subset $X_{\alpha}$ of $X$ such that

(i) $X_{\alpha}$ is the countable union of open sets, each of which is connected, hereditarily separable and has compact closure, and

(ii) $\operatorname{cl}\left(\cup_{\beta<\alpha} X_{\beta}\right) \subset X_{\alpha}$.

Since $X$ is locally compact and locally connected we can clearly make such choices unless there is an $\alpha<\omega_{1}$ such that $\operatorname{cl}\left(\cup_{\beta<\alpha} X_{\beta}\right)$ is not Lindelöf.

Assume that $\lambda<\omega_{1}$ and that $X_{\alpha}$ has been constructed for $\alpha<\lambda$. Let $C=$ $\operatorname{cl}\left(\cup_{\alpha<\lambda} X_{\alpha}\right) . C$ is separable. Now suppose that $C$ is not hereditarily separable. Then there exists $S=\left\{x_{\beta}\right\}_{\beta<\omega_{1}} \subset C$ such that if $\beta<\omega_{1}$ then $x_{\beta} \notin \operatorname{cl}\left\{x_{\alpha}\right\}_{\alpha<\beta}$. For each $\beta<\omega_{1}$ let $U_{\beta}$ denote a hereditarily separable open set in $X$ such that $x_{\beta} \in U_{\beta}$ and $U_{\beta} \cap \operatorname{cl}\left\{x_{\alpha}\right\}_{\alpha<\beta}=\varnothing$. Since $S$ is not separable, no countable subcollection of the $U_{\beta}$ 's covers $S$. So there is an uncountable subset $A$ of $\omega_{1}$ such that, if $\beta<\alpha$ in $A$, then $x_{\alpha} \notin U_{\beta}$. Since $X$ is perfect there is a sequence $\left\{V_{n}\right\}_{n \in \omega}$ of open sets such that $\cap_{n=1}^{\infty} V_{n}=\left(C-\cup_{\alpha \in A} U_{\alpha}\right)$. For some $n \in \omega, A_{n}=\left\{\alpha \in A \mid x_{\alpha} \notin V_{n}\right\}$ is uncountable. Observe that $S_{n}=\left\{x_{\alpha} \mid \alpha \in A_{n}\right\}$ is a closed discrete subset of $X$. By Theorem 4 we can separate the points of $S_{n}$ with a disjoint collection of open sets. But this contradicts the fact that $C$ is separable. We conclude that $C$ is hereditarily separable. Since $C$ is locally compact, Theorem 1 implies that $C$ is hereditarily Lindelöf as well. Therefore we can construct $X$ satisfying (i) and (ii).

Observe that $\cup_{\alpha<\omega_{1}} X_{\alpha}$ is both open and closed, and since we are assuming $X$ is connected, $\cup_{\alpha<\omega_{1}} X_{\alpha}=X .\left(\cup_{\alpha<\omega_{1}} X_{\alpha}\right.$ is open by definition. $X$ is perfect and locally compact and therefore is first countable. Since $\operatorname{cl}\left(\cup_{\beta<\alpha} X_{\beta}\right) \subset X_{\alpha}$ for $\alpha<\omega_{1}$, there can be no points of $X$ in $\operatorname{cl}\left(\cup_{\alpha<\omega_{1}} X_{\alpha}\right)-\cup_{\alpha<\omega_{1}} X_{\alpha}$. $)$

So we have a perfectly normal, locally compact and locally connected space $X=\cup_{\alpha<\omega_{1}} X_{\alpha}$ where each $X_{\alpha}$ is open, hereditarily Lindelöf and $\operatorname{cl}\left(\cup_{\beta<\alpha} X_{\beta}\right) \subset$ $X_{\alpha}$. We let $X_{\alpha}^{\prime}=X_{\alpha}-\cup_{\beta<\alpha} X_{\beta}$. In order to show that $X$ is subparacompact we use the characterization of perfect subparacompactness given in Theorem 5 .

Let $\left\{W_{\beta}\right\}_{\beta<\gamma}$ be a well-ordered open cover of $X$. Since each $X_{\alpha}$ is perfect and subparacompact, for each $\alpha<\omega_{1}$ there is a sequence $\left\{\mathcal{U}_{\alpha n}\right\}_{n \in \omega}$ of open covers of $X_{\alpha}$ having the property described in Theorem 5 with respect to the open cover $\left\{W_{\beta} \cap X_{\alpha}\right\}_{\beta<\gamma}$ of $X_{\alpha}$. We may assume that $\mathscr{U}_{\alpha(n+1)}$ refines $\mathscr{U}_{\alpha n}$ for $n \in \omega$ and $\alpha<\omega_{1}$.

For each $\alpha<\omega_{1}$ we can choose by induction a sequence $\left\{\mathcal{K}_{\alpha n}\right\}_{n \in \omega}$ having the following properties. 
(a) $\mathcal{K}_{\alpha n}$ is a countable collection of open sets covering $X_{\alpha}^{\prime}$ and refining $\mathcal{Q}_{\alpha n}$,

(b) if $K \in \mathscr{K}_{\alpha n}, \operatorname{cl}(K)$ is compact, $\operatorname{cl}(K) \subset X_{\alpha}$ and $K \cap X_{\alpha}^{\prime} \neq \varnothing$, and

(c) if $x \in X_{\alpha}^{\prime}$ and $\mathscr{F}$ is a finite subcollection of $U\left\{\mathscr{K}_{\beta j} \mid \beta<\alpha\right.$ and $\left.j \in \omega\right\}$ then there are infinitely many distinct elements of $\mathcal{K}_{\alpha n}$ containing $x$ and not intersecting $\operatorname{cl}(\cup \mathscr{F})$.

Note that since $X$ is locally connected and normal, there are uncountably many distinct open sets containing a given point and lying within a given neighborhood. This, together with the fact that $X_{\alpha}$ is Lindelöf, allows us to easily construct $\mathcal{K}_{\alpha n}$ satisfying (c).

Let $\mathcal{K}_{\alpha}=\cup_{n \in \omega} \mathcal{K}_{\alpha n}$; let $\mathcal{K}_{=}=\cup_{\alpha<\omega_{1}} \mathcal{K}_{\alpha}$. Note that $\left|\mathcal{K}_{\alpha}\right|=\omega$ and $\left|K_{K}\right|=\omega_{1}$. For $K \in \mathcal{K}_{\alpha}$, define $g(K)=\alpha$; observe that $g(K)=\sup \left\{\beta \mid K \cap X_{\beta}^{\prime} \neq \varnothing\right\}$.

Define $P$ to be the set of all functions $f$ such that

(1) the domain of $f$, called $D(f)$, is a finite subset of $\mathcal{K}$,

(2) the range of $f$, called $R(f)$, is a subset of $\omega$, and

(3) if $H, K \in D(f), g(H)<g(K)$, and $H \cap K \cap X_{g(H)}^{\prime} \neq \varnothing$, then $f(H)>f(K)$.

Partially order $P$ by defining $f \leqslant g$ provided $g$ extends $f$.

If $K \in \mathcal{K}_{\alpha n}$, define $F_{K n}=\left\{f \in P \mid D(f) \cap \mathcal{K}_{\alpha n} \cap\{H \mid f(H)>n\}\right.$ covers $K \cap$ $\left.X_{\alpha}^{\prime}\right\}$. Clearly, $F_{K n}$ is open in $(P, \leqslant)$. We will prove that $F_{K n}$ is dense. Suppose $f \in P$. Since $\bar{K} \cap X_{\alpha}^{\prime}$ is compact and $\mathscr{K}_{\alpha n}$ has property (c), we can choose a finite collection $\mathcal{G} \subset \mathcal{K}_{\alpha n}$ such that $\mathcal{G}$ covers $K \cap X_{\alpha}^{\prime}, \mathcal{G} \cap D(f)=\varnothing$, and if $G \in \mathcal{G}$ and $J \in D(f) \cap\{H \in \mathcal{K} \mid g(H)<\alpha\}$ then $G \cap \bar{J}=\varnothing$. Let

$$
m=\max \{n, \max \{f(H) \mid H \in D(f)\}\} .
$$

We choose $h \in P$ such that $D(h)=D(f) \cup \mathcal{G}, h \uparrow D(f)=f$, and if $G \in \mathcal{G} f(G)=$ $m$. Since $h$ extends $f$ and $h \in F_{K n}, F_{K n}$ is dense in $(P,<)$.

The proof that $(P,<)$ is ccc is identical to the proof given by Rudin.

Since $(P, \leqslant)$ is ccc, $\left\{F_{K n}: K \in \mathcal{K}, n \in \omega\right\}$ has cardinality $\omega_{1}$, and each $F_{K n}$ is open and dense in $(P, \leqslant)$, by $(\mathrm{MA}+\neg \mathrm{CH})$ there is a generic $G \subset P$ which intersects every $F_{K n}$. If $f$ and $f^{\prime}$ belong to $G$ there is an $h \in P$ such that $h$ extends both $f$ and $f^{\prime}$. We use this $G$ to find a sequence $\left\{\mathscr{Q}_{n}\right\}_{n \in \omega}$ of covers of $X$ satisfying Theorem 5 with respect to the open cover $\left\{W_{\beta}\right\}_{\beta<\gamma}$.

Let $\mathscr{K}^{\prime}=\{K \in \mathscr{K} \mid K \in D(f)$ for some $f \in G\}$. Note that if $f$ and $f^{\prime}$ belong to $G$ and $K \in D(f) \cap D\left(f^{\prime}\right)$ then $f(K)=f^{\prime}(K)$. We define a function $F$ : $\mathcal{K}^{\prime} \rightarrow \omega$ by $F(K)=f(K)$ where $f \in G$ and $K \in D(f)$. Let $D_{n}=\{K \in \mathcal{K} \mid F(K)>n\}$.

For $n \in \omega, \alpha<\omega_{1}$, and $x \in X_{\alpha}^{\prime}$, choose $U_{x n} \in D_{n} \cap \mathscr{K}_{\alpha n}$ with $x \in U_{x n}$. Such a $U_{x n}$ exists since if $x \in X_{\alpha}^{\prime}$ there is a $K \in \mathcal{K}_{\alpha n}$ containing $x$ and $G \cap F_{K n} \neq \varnothing$. Let $U_{n}=\left\{U_{x n} \mid x \in X\right\}$.

We claim that $\left\{\mathscr{U}_{n}\right\}_{n \in \omega}$ witnesses the fact that $X$ is (perfectly) subparacompact. To see this, suppose $x \in X$ and $\beta<\omega_{1}$ is the first ordinal such that $x \in W_{\beta}$. There is an $\alpha<\omega_{1}$ such that $x \in X_{\alpha}^{\prime}$. By (a) there is an $m \in \omega$ such that $\operatorname{st}\left(x, \mathscr{K}_{\alpha} m\right) \subset$ $W_{\beta}$. Choose $K_{x} \in \mathcal{K}_{\alpha} \cap \mathcal{K}^{\prime}$ such that $x \in K_{x}$. Let $n=\max \left\{m, F\left(K_{x}\right)\right\}$. We show that st $\left(x, \mathscr{Q}_{n}\right) \subset W_{\beta}$.

Suppose that $x \in U_{y n} \in \mathcal{U}_{n}$. Then $y \in X_{\delta}^{\prime}$ for some $\delta<\omega_{1}$ and $U_{y n} \in D_{n} \cap$ $\mathcal{K}_{\delta \boldsymbol{n}}$.

Case (i). $\alpha=\delta$. Since $\mathscr{\vartheta}_{\alpha n}$ refines $\mathscr{U}_{\alpha m}, \operatorname{st}\left(x, \mathcal{K}_{\alpha n}\right) \subset W_{\beta}$ and $U_{y n} \subset \operatorname{st}\left(x, \mathcal{K}_{\alpha n}\right)$. 
Case (ii). $\alpha>\delta$. Since $U_{y n} \in \mathscr{K}_{\delta n}$ implies that $U_{y n} \subset X_{\delta}^{\prime}, \alpha>\delta$ contradicts the fact that $x \in U_{y n}$.

Case (iii). $\alpha<\delta$. Since $U_{y n} \in D_{n}$ we know that $F\left(U_{y n}\right)>n>F\left(K_{x}\right)$. However since $K_{x}$ and $U_{y n}$ are both in $\mathcal{K}^{\prime}$ and $G$ is generic in $(P,<)$, there is an $f \in P$ such that $H_{x}$ and $U_{y n}$ are in $D(f)$. Since $\alpha=g\left(K_{x}\right)$ and $\delta=g\left(U_{y n}\right)$ and $X_{\alpha}^{\prime} \cap K_{x} \cap U_{y n}$ $\neq \varnothing, \alpha<\delta$ gives a contradiction to (3).

Thus the sequence $\left\{\mathcal{Q}_{n}\right\}_{n \in \omega}$ has the desired property. By Theorem $5, X$ is subparacompact.

Corollary to TheOREM 6. If $(\mathrm{MA}+\neg \mathrm{CH})$, then every component of a perfectly normal, locally compact, locally connected space is Lindelöf.

This follows from the fact that every locally compact, paracompact space is the free union of $\sigma$-compact spaces.

\section{REFERENCES}

[A, Z] K. Alster and P. L. Zenor, On the collectionwise normality of generalized manifolds, Topology Proceedings, Vol. I (Conf. Auburn Univ., Auburn, Ala., 1976), Math Dept., Auburn Univ., Auburn, Ala., 1977, pp. 125-127.

[C, Z] J. Chaber and P. L. Zenor, On perfect subparacompactness and a metrization theorem for Moore spaces, Topology Proceedings, Vol. II (Conf. Auburn Univ., Auburn, Ala., 1977), Math. Dept., Auburn Univ., Auburn, Ala., 1978, pp. 401-407.

[J] I. Juhasz, Cardinal functions in topology, Math. Centre Tract, no. 34, Mathematisch Centrum, Amsterdam, 1971.

[R, Z] G. M. Reed and P. L. Zenor, Metrization of Moore spaces and generalized manifolds, Fund. Math. 91 (1976), 203-210.

[Ru] M. E. Rudin, The undecidability of the existence of a perfectly normal nonmetrizable manifold, preprint, 1978.

[Ru, Z] M. E. Rudin and P. L. Zenor, A perfectly normal nonmetrizable manifold, Houston J. Math. 2 (1976), 129-134.

[S] Z. Szentmiklossy, S-spaces and L-spaces under Martin's Axiom, preprint, 1978.

Department of Mathematics, Auburn University, Auburn, Alabama 36830 